\title{
Hard-sphere radial distribution function again
}

Andrij Trokhymchuk

Douglas Henderson

Ivo Nezbeda

Jan Jirsak

Follow this and additional works at: https://scholarsarchive.byu.edu/facpub

Part of the Biochemistry Commons, and the Chemistry Commons

\section{Original Publication Citation}

Trokhymchuk, Andrij, Ivo Nezbeda, Jan Jirsak, and Douglas Henderson."'Hard-sphere radial distribution function again." The Journal of Chemical Physics 123 (25).

\section{BYU ScholarsArchive Citation}

Trokhymchuk, Andrij; Henderson, Douglas; Nezbeda, Ivo; and Jirsak, Jan, "Hard-sphere radial distribution function again" (2005). Faculty Publications. 360.

https://scholarsarchive.byu.edu/facpub/360 


\title{
Hard-sphere radial distribution function again
}

\author{
Andrij Trokhymchuk ${ }^{\mathrm{a})}$ \\ Department of Chemistry and Biochemistry, Brigham Young University, Provo, Utah 84602 \\ Ivo Nezbeda \\ E. Hála Laboratory of Thermodynamics, Institute of Chemical Process Fundamentals, \\ Academy of Science, 16502 Prague 6, Suchdol, Czech Republic and Department of Physics, \\ J. E. Purkyně University, 40096 Ústí nad Labem, Czech Republic \\ Jan Jirsák \\ E. Hála Laboratory of Thermodynamics, Institute of Chemical Process Fundamentals, \\ Academy of Science, 16502 Prague 6, Suchdol, Czech Republic \\ Douglas Henderson \\ Department of Chemistry and Biochemistry, Brigham Young University, Provo, Utah 84602
}

(Received 22 April 2005; accepted 1 June 2005; published online 18 July 2005)

A theoretically based closed-form analytical equation for the radial distribution function, $g(r)$, of a fluid of hard spheres is presented and used to obtain an accurate analytic representation. The method makes use of an analytic expression for the short- and long-range behaviors of $g(r)$, both obtained from the Percus-Yevick equation, in combination with the thermodynamic consistency constraint. Physical arguments then leave only three parameters in the equation of $g(r)$ that are to be solved numerically, whereas all remaining ones are taken from the analytical solution of the Percus-Yevick equation. (C) 2005 American Institute of Physics. [DOI: 10.1063/1.1979488]

\section{INTRODUCTION}

Because of its unique role in modern theories of classical fluids, the fluid of hard spheres (HSs) has undoubtedly been by far the most intensively studied system of all model fluids. Thanks to numerous computer simulation studies (for a review see Refs. 1 and 2 and references in Ref. 3 for recent works), both the structural and thermodynamic properties of the HS fluid are known to a high degree of accuracy. Yet, accurate data for some fundamental functions that are difficult to simulate, as, e.g., the direct correlation function (DCF) and bridge function, are still missing. Thus, the HS fluid has kept drawing the attention both of simulators and theoreticians (e.g., for a recently developed general method to evaluate elementary diagrams, and, hence, the bridge function at low densities, see Ref. 4).

What has, however, made the HS fluid particularly attractive and important is the availability of a reasonably accurate, but still an approximate, theoretical result for the radial distribution function (RDF), $g(r)$. Wertheim ${ }^{5}$ and Thiele ${ }^{6}$ found, using different methods, an analytic solution of the Percus-Yevick (PY) integral equation. Wertheim obtained $g(r)$ in the form of its Laplace transform, $\mathcal{G}=\mathcal{L}\{g\}$, and in the form of an expansion. Smith and Henderson ${ }^{7}$ provided analytic formulas for the inverse transform $g=\mathcal{L}^{-1}\{\mathcal{G}\}$, and Nezbeda ${ }^{8}$ provided then an analytic result directly for $g(r)$. The latter method was then used by Perram ${ }^{9}$ to develop a code for generating $g(r)$ numerically. This was also rederived again recently by Chang and Sandler. ${ }^{10}$

\footnotetext{
a) Author to whom correspondence should be addressed; permanent address: Institute for Condensed Matter Physics, National Academy of Sciences of Ukraine, Lviv 79011, Ukraine. Electronic mail: adt@1cmp.lviv.ua
}

All the above-mentioned theoretical results have provided the basis for a number of later empirical improvements aiming at more accurate and/or more manageable expressions of $g(r)$. Another approach consists of a direct empirical parametrization of accurate simulation data, see, e.g., the parametrization result of Verlet and Weiss ${ }^{11}$ and the result for the bridge function by Malijevský and Labík. ${ }^{12}$ All these results have considerably contributed to the development of theories of liquids. However, their application is limited. Because of their purely empirical nature, they can be used safely only over the range of existing simulation data and, thus, do not allow any guaranteed prediction. This drawback becomes especially important for systems/conditions where simulations may fail (e.g., mixtures of HS of a large diameter ratio) and justifies therefore continuing theoretical efforts.

From the theoretical point of view, the most popular and useful route to $g(r)$ is the Ornstein-Zernike (OZ) integral equation supplemented by a closure that establishes the bridge function. ${ }^{13}$ There are two widely used theoretically based closures: hypernetted chain (HNC) and PY, ${ }^{13}$ most of the other closures used so far represent only empirical modifications with the goal of improving the accuracy of the resulting $g(r) .{ }^{14,15}$ As an alternative to such empirical modifications of closures one may use a closure with unspecified parameters and impose certain constraints. This route was followed in a series of papers by Lee ${ }^{16-18}$ in his selfconsistent integral equation theory based on an extended Verlet closure. ${ }^{14}$ Nonetheless, neither of the above methods have been successful so far and their results are inferior to empirical parametrizations. A third possibility towards obtaining a nonempirical analytic representation of $g(r)$ is to assume a certain theoretically justified functional form for $g(r)$ (e.g., a 
result of the solution of $\mathrm{OZ}$ equation) with adjustable parameters and impose then certain constraints (e.g., thermodynamic consistency) to determine these parameters. This route is pursued in this present study.

The purpose of this paper is twofold, both theoretical and practical. From the theoretical point of view, the goal is to obtain an analytic and an accurate functional representation of $g(r)$, without resorting to extensive computer simulation data, using the above-mentioned ideology. From a practical point of view, we should obtain an accurate expression of $g(r)$ that could be used later in various applications; particularly appealing would be its extension to mixtures of hard spheres of a large diameter ratio. We propose here a method that is based on two analytic results: (i) the asymptotic behavior of $g(r)$ for $r \rightarrow+\infty$, and (ii) the analytic solution of the PY equation in the contact region $\sigma \leqslant r \leqslant 2 \sigma$, where $\sigma$ is the hard-core diameter. Unlike purely empirical methods that usually require extensive computer simulation data for $g(r)$ over the entire physically relevant range of densities and particle-particle separations, the only experimental input required in the proposed method is the so-called contact value of the RDF, i.e., value $g_{\sigma}$ of $g(r)$ at $r=\sigma$, and the only physical constraint imposed is thermodynamic consistency ${ }^{13}$ of the pressure, $p$, obtained from the virial equation,

$$
\frac{p}{\rho k_{B} T}=1+4 \eta g_{\sigma}
$$

and the compressibility equation,

$$
k_{B} T\left(\frac{\partial \rho}{\partial p}\right)_{T}=1+4 \pi \rho \int[g(r)-1] r^{2} d r
$$

In Eqs. (1) and (2) the notation $\rho$ corresponds to the number density of HS fluid while $\eta=\pi \rho \sigma^{3} / 6$ is the packing fraction, $T$ is the temperature, and $k_{B}$ is the Boltzmann constant. Other possible constraints, as e.g., the zero-separation theorems, ${ }^{16-18}$ are used only a posteriori to check and assess the obtained result. Although the starting expression of $g(r)$ contains a number of parameters, it turns out that most of them take on values given by the solution of the PY equation, leaving only three parameters to be adjusted according to the imposed constraints. After providing a theoretical background in Sec. II, we explain in detail the used method in Sec. III, and discuss the results in Sec. IV.

\section{BASIC DEFINITIONS AND THEORETICAL BACKGROUND}

The RDF satisfies the OZ equation, ${ }^{13,19}$

$$
\gamma(r) \equiv g(r)-c(r)-1=\rho \int\left[\gamma\left(\mathbf{r}^{\prime}\right)+c\left(\mathbf{r}^{\prime}\right)\right] c\left(\left|\mathbf{r}-\mathbf{r}^{\prime}\right|\right) d \mathbf{r}^{\prime},
$$

where $c(r)$ is the DCF, and $\rho$ is the number density. This equation contains two unknowns: $\gamma(r)$ and $c(r)$. Thus, to make solution possible, one must supplement this equation with a closure. For the HS fluid defined by the pair interaction potential,

$$
\begin{aligned}
u(r) & =\infty \text { for } r<\sigma \\
& =0 \text { for } r>\sigma,
\end{aligned}
$$

there is one exact condition,

$$
g(r)=0 \text { for } r<\sigma .
$$

The other condition is an approximate closure. The most common choice is the PY approximation, which reads as

$$
c^{\mathrm{PY}}(r) \equiv 0 \quad \text { for } r>\sigma .
$$

In the above equations $\sigma$ denotes the hard-core diameter that, if necessary, may be set to unity without loss of generality.

The exact solution of the PY approximation for the RDF was found independently by Wertheim ${ }^{5}$ and Thiele. ${ }^{6}$ Wertheim solved the PY equation for the Laplace transform $\mathcal{G}^{\mathrm{PY}}(s)=\mathcal{L}\left\{g^{\mathrm{PY}}(r)\right\}$ and this solution will be used for our purposes. It reads as

$$
g^{\mathrm{PY}}(r)=\mathcal{L}^{-1}\left\{\mathcal{G}^{\mathrm{PY}}(s)\right\}=\frac{1}{2 \pi i r} \int_{\delta-i \infty}^{\delta+i \infty} \frac{s L(s) e^{s r} d s}{12 \eta\left[S(s) e^{s}+L(s)\right]},
$$

where

$$
S(s)=(1-\eta) s^{3}+6 \eta(1-\eta) s^{2}+18 \eta^{2} s-12 \eta(1+2 \eta),
$$

$$
L(s)=12 \eta\left(1+\frac{1}{2} \eta\right) s+12 \eta(1+2 \eta) .
$$

From the properties of the integrand in Eq. (7) it follows that $g^{\mathrm{PY}}(r)=0$ for $r<\sigma$, as required by Eq. (5). For $r \geqslant \sigma$, the integral (7) should be inverted. The inversion can be performed by means of the residue theorem in the standard way,

$$
g^{\mathrm{PY}}(r)=\sum_{n=0}^{\infty} g_{n}^{\mathrm{res}}(r) \quad \text { for } r \geqslant \sigma,
$$

where $g_{n}^{\text {res }}(r)$ is the residue of $\mathcal{G}^{\mathrm{PY}}(s)$ at the roots $s_{n}$ of the denominator $L(s)+S(s) e^{s}$. The residue at $s_{0}=0$ contributes 1 to $g^{\mathrm{PY}}(r)$. The other roots, $s_{n}$, occur as complex-conjugate pairs. Among them there exists a pair $s_{1,2}=\kappa_{o} \pm i \omega_{o}$ that is closest to the real axis and which determines the asymptotic behavior of $g^{\mathrm{PY}}(r)-1$ for large $r$. Thus, one obtains ${ }^{20}$

$$
g^{\mathrm{PY}}(r)=1+\frac{C_{o}}{r} \cos \left(\omega_{o} r+\delta_{o}\right) e^{-\kappa_{o} r} \quad \text { for } r \rightarrow \infty,
$$

where $C_{o}$ and $\delta_{o}$ are the density-dependent coefficients that are uniquely defined once $\kappa_{o}$ and $\omega_{o}$ are known. In general, Eq. (10) is a sum over an infinite number of roots $s_{n}$ of $L(s)+S(s) e^{s}$ with each $g_{n}^{\text {res }}(r)$ being continuous and contributing to $g^{\mathrm{PY}}(r)$ for all $r \geqslant \sigma$. In order to obtain $g^{\mathrm{PY}}(r)$ in a closed form for a given $r$, Wertheim used an expansion of the denominator of Eq. (7) in powers of $L(s) / S(s)$ so that $g^{\mathrm{PY}}(r)$ for $r \geqslant \sigma$ assumes the form 

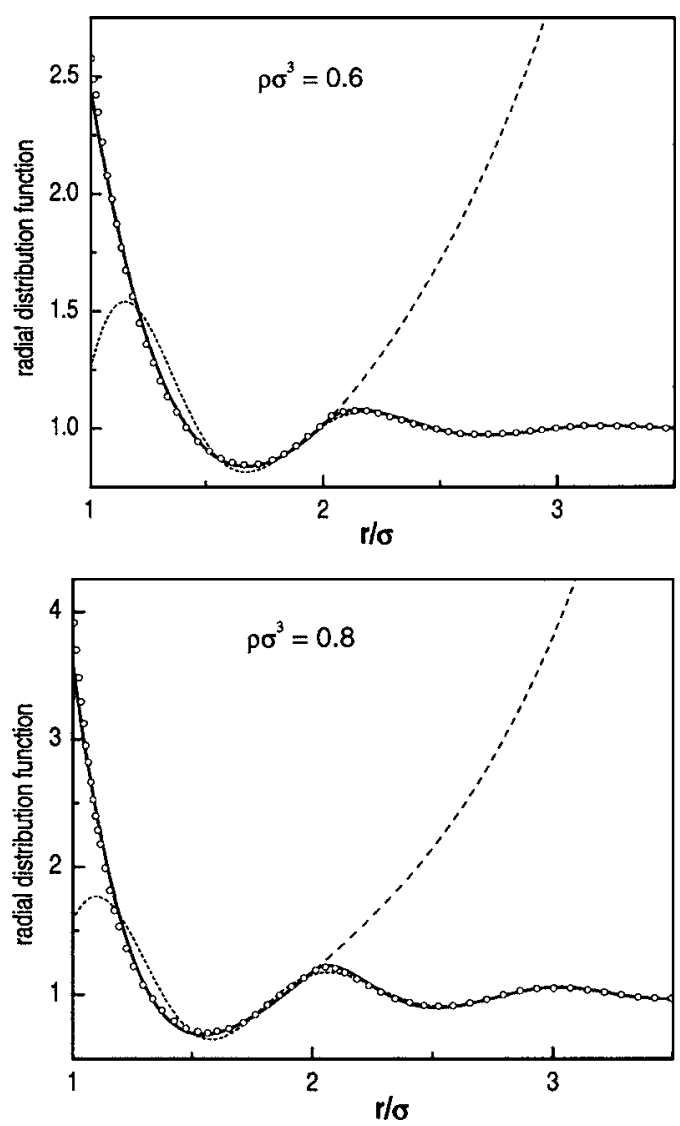

FIG. 1. Comparison of $g^{\operatorname{dep}}(r)$ (dashed lines) and $g^{\text {str }}(r)$ (short dashed lines) with $g^{\mathrm{PY}}(r)$ (solid lines). The symbols denote MC data.

$$
g^{\mathrm{PY}}(r)=\sum_{i=1}^{\infty} g_{i}^{\mathrm{zone}}(r) \quad \text { for } r \geqslant \sigma,
$$

where $g_{i}^{\text {zone }}(r)$ is the sum of the residue of $\mathcal{G}^{\mathrm{PY}}(s)$ at three roots of $S(s)$ of the order $i: s_{0}=\mu_{o}$ and $s_{1,2}=\alpha_{o} \pm i \beta_{o}$. Equation (12) is a sum over an infinite number of the distance intervals (zones) with each individual $g_{i}^{\text {zone }}(r)$ behaving as a stepwise function $g_{i}^{\text {zone }}(r)=0$ for $r / \sigma<i$, i.e., contributing to $g^{\mathrm{PY}}(r)$ only for $r / \sigma \geqslant i$. For the first zone, $1 \leqslant r / \sigma \leqslant 2$, there is only one term in Eq. (12) and the result is explicit in the form $^{5,7,8}$

$$
\begin{aligned}
g^{\mathrm{PY}}(r)= & \frac{A_{o}}{r} e^{\mu_{o}[r-\sigma]}+\frac{B_{o}}{r} \cos \left(\beta_{o}[r-\sigma]\right. \\
& \left.+\gamma_{o}\right) e^{\alpha_{o}[r-\sigma]} \text { for } \sigma \leqslant r \leqslant 2 \sigma,
\end{aligned}
$$

where $A_{o}, B_{o}$, and $\gamma_{o}$ are the density-dependent coefficients that are uniquely defined once $\mu_{o}, \alpha_{o}$, and $\beta_{o}$ are known. In Eqs. (11) and (13) and henceforth in this paper, the parameters with subscript "o" are those that follow from the PY theory and are assumed to be known.

The above solution of the PY equation for the fluid of hard spheres has become a keystone result in the molecular physics of liquids. In fact, it is one of only a few analytic theoretical results in theories of the liquid state. As a result of an approximate theory, it suffers from certain defects. For the PY theory, these may be summarized as follows: ${ }^{11}$ (i) $g^{\mathrm{PY}}(r)$ is incorrect at the contact, $r=\sigma$, (ii) $g^{\mathrm{PY}}(r)$ oscillates slightly out of phase compared to the Monte Carlo (MC) data, and (iii) the thermodynamics obtained from Eqs. (1) and (2) are not consistent. Nonetheless, these inaccuracies are not so severe and the above solution provides a useful springboard for the development of a better representation.

\section{PARAMETRIZED RADIAL DISTRIBUTION FUNCTION}

\section{A. General considerations}

In Fig. 1 we compare the PY result for hard-sphere RDF and its asymptotic forms, Eqs. (11) and (13), respectively, with simulation data for two typical liquid densities. The fact that the single short-distance term given by the simple expression (13) fits $g^{\mathrm{PY}}(r)$ well throughout the first distance zone, $1 \leqslant r / \sigma \leqslant 2$, is not surprising. However, it is surprising that just the single long-range asymptotic term, Eq. (11), fits $g^{\mathrm{PY}}(r)$ remarkably well also for intermediate distances starting already from the second distance zone, $r / \sigma>2$. Moreover, both these terms have a simple functional form and it is therefore tempting to write $g(r)$ over the entire range of distances as a combination of these two terms.

The considered analytic representation of $g(r)$ is thus given by a superposition of the two functional forms given by Eqs. (11) and (13),

$$
\begin{aligned}
g(r) & =0 \quad \text { for } r<\sigma \\
& =g^{\operatorname{dep}}(r) \quad \text { for } \sigma \leqslant r \leqslant r^{\star} \\
& =g^{\operatorname{str}}(r) \quad \text { for } r \geqslant r^{\star} .
\end{aligned}
$$

Here

$$
g^{\operatorname{dep}}(r)=\frac{A}{r} e^{\mu[r-\sigma]}+\frac{B}{r} \cos (\beta[r-\sigma]+\gamma) e^{\alpha[r-\sigma]},
$$

and will be referred to as a depletion part of the RDF, and

$$
g^{\operatorname{str}}(r)=1+\frac{C}{r} \cos (\omega r+\delta) e^{-\kappa r},
$$

referred to as a structural part of the RDF; $r^{*}$ denotes the distance where these two parts merge. Equations (14)-(16) contain altogether 11 unknown parameters: ten in the functional expression and the 11th parameter is the separation $r^{*}$. The problem we must solve now is to find such a set of parameters that the RDF given by Eq. (14) reproduces as accurately as possible the known experimental (computer simulation) data.

It is evident that, with respect to the approximate nature and functional form of Eqs. (14)-(16), the solution cannot be unique. One straightforward way is to use brute force and employ a multiparameter minimization routine to find a best fit of $g(r)$ to the known simulation data. However, such a purely empirical approach, pursued, e.g., by Malijevský and Labík in their parameterization of the bridge function, ${ }^{12}$ brings little further progress and understanding. Our goal is to have an equation for $g(r)$ which is accurate and simultaneously reflects as much physics as possible, thus reducing empirical fitting to a minimum. The question to what extent this goal can be achieved with $g(r)$ in the form of Eq. (14) we address later in the discussion part of this paper. 
There is a number of well-known physical conditions that may be imposed on $g(r)$. Two obvious and most common conditions are to require that (i) $g(r)$ yield an accurate equation of state (pressure), and (ii) the pressure calculated via the virial and compressibility routes be identical (thermodynamic consistency). It is also evident that (iii) $g(r)$ must be continuous and (iv) smooth at $r^{*}$. The last two conditions require some comments. From the discussion of the shortrange and asymptotic parts of the PY solution in Sec. II, and from Fig. 1 it follows that $g(r)$, given by Eq. (14), may be expected to perform accurately in the region of the nearest neighbors, $r \leqq r_{m}$ where $r_{m}$ is the location of the first minimum $\left(r_{m} / \sigma \approx 1.5-1.9\right.$ depending on the density), and beyond the first shell, $r / \sigma \gtrsim 2$. The parameter $r^{*}$ should thus lie somewhere between these two bounds and its choice seems crucial for the success of the approach. It might be treated as a free adjustable parameter but this seems rather cumbersome. Our choice for the merging distance is $r^{*} \equiv r_{m}$ that, besides a desired physical argument (an integral of $r^{2} g(r)$ over $r$ from $\sigma$ to $r_{m}$ is directly related to the first coordination number), imposes naturally an extra constraint on $g(r)$ at $r$ $=r^{*}$. Namely, it says that $g(r)$ must be not only continuous at $r=r^{*}$ but also smooth and at a minimum, i.e., with a fixed and zero slope. Due to this, the parameter $r^{*}$ can be solved in a self-consistent manner with all other parameters of $g(r)$ and compared against experimental (computer simulation) data for the position of the first minimum of the HS fluid RDF.

The above conditions and arguments constitute the following set of equations:

$$
\begin{aligned}
& g^{\mathrm{dep}}\left(r=\sigma^{+}\right)=g_{\sigma}^{\text {expt }}, \\
& K_{T} \equiv k_{B} T\left(\frac{\partial \rho}{\partial p}\right)_{T}=1+4 \pi \rho \int_{0}^{\infty}[g(r)-1] r^{2} d r \\
& =\left[1+8 \eta g_{\sigma}^{\mathrm{expt}}+4 \eta^{2}\left(\frac{\partial g_{\sigma}^{\text {expt }}}{\partial \eta}\right)\right]^{-1}, \\
& g^{\mathrm{dep}}\left(r=r^{*}\right)=g_{m} \text { and } g^{\mathrm{str}}\left(r=r^{*}\right)=g_{m}, \\
& {\left[\frac{d}{d r} g^{\mathrm{dep}}(r)\right]_{r=r^{*}}=0 \text { and }\left[\frac{d}{d r} g^{\mathrm{str}}(r)\right]_{r=r^{*}}=0,}
\end{aligned}
$$

where the superscript "expt" denotes experimental (computer simulation) data and $g_{m}$ is the RDF value at the distance of the first minimum $r=r_{m}$. We note that the last two equations (19) and (20) are specified for our choice of the merging distance $r^{*} \equiv r_{m}$. This prevents their application at low densities $\rho$ where $g(r)$ does not have minima, being monotonic for all distances $r$. Because of this we limit the present study for densities $\rho \sigma^{3} \geqslant 0.2$. However, the low-density region still can be studied in a similar way by assuming, for instance, that the merging distance is at the end of the first distance zone, i.e., $r^{*}=2 \sigma$ and by using instead of the pair of Eqs. (19) and (20) the single constraint $g^{\mathrm{dep}}\left(r=r^{*}\right)=g^{\mathrm{str}}\left(r=r^{*}\right)$.

A closer examination of Eqs. (17)-(20) shows that not all the unknown parameters in equation for $g(r)$ are independent. In particular, using constraints (17) and (19), the parameters $A$ and $B$ of the depletion part of $g(r)$ can be uniquely expressed through $\alpha, \beta, \gamma, \mu$, the position of the first minimum $r^{*} \equiv r_{m}$, the minimum value $g_{m}$, and the known contact value $g_{\sigma}^{\text {expt }}$, as follows:

$B$

$$
=\frac{g_{m}-\left(\sigma g_{\sigma}^{\operatorname{expt}} / r^{*}\right) \exp \mu\left[r^{*}-\sigma\right]}{\cos \left(\beta\left[r^{*}-\sigma\right]+\gamma\right) \exp \alpha\left[r^{*}-\sigma\right]-\cos \gamma \exp \mu\left[r^{*}-\sigma\right]} r^{*},
$$

and

$$
A=\sigma g_{\sigma}^{\mathrm{expt}}-B \cos \gamma .
$$

In a similar way constraints (19) and (20) allow us to express the parameters $\delta$ and $C$ of the structural part of $g(r)$ through $\omega, \kappa$, and $r^{*}$, and $g_{m}$ in the form,

$$
\delta=-\omega r^{*}-\arctan \frac{\kappa r^{*}+1}{\omega r^{*}},
$$

and

$$
C=\frac{r^{*}\left[g_{m}-1\right] \exp \kappa r^{*}}{\cos \left(\omega r^{*}+\delta\right)} .
$$

The remaining six parameters $\alpha, \beta, \gamma, \mu, \omega$, and $\kappa$ as well as the position of the first minimum $r^{*} \equiv r_{m}$ and the minimum value $g_{m}$ are obtained from the solution of the above set of equations (17)-(20).

To solve this set of equations, the experimental value of $g_{\sigma}^{\text {expt }}$ is required. For this purpose we use the latest result reported by Kolafa et al., ${ }^{3}$ who provides $g_{\sigma}^{\text {expt }}$ in the form,

$$
g_{\sigma}^{\text {expt }}=\frac{1}{4 \eta}\left(\frac{1+\eta+\eta^{2}-2 / 3 \eta^{3}-2 / 3 \eta^{4}}{(1-\eta)^{3}}-1\right) .
$$

The isothermal compressibility, $K_{T}$, is evaluated from $g_{\sigma}^{\text {expt}}$; for convenience and its later use we give this result here,

$$
K_{T}^{\text {expt }}=\frac{3(1-\eta)^{4}}{3+12 \eta+12 \eta^{2}-8 \eta^{3}-8 \eta^{4}+4 \eta^{5}} .
$$

We wish to recall that although in the discussion of our results we are using reduced density $\rho \sigma^{3}$, the equations are written in terms of packing fraction, $\eta=\pi \rho \sigma^{3} / 6$, that is more convenient for the HS fluid.

\section{B. Results}

It is known that the fitting of nonlinear expressions is sensitive to input values and different solutions can be found with different inputs. To minimize this arbitrariness, we impose the condition that in the limit of low densities all parameters approach the PY values. Using then a numerical algorithm based on the AMOEBA routine ${ }^{21}$ we have found that the values of four parameters, namely, $\kappa, \omega, \gamma$, and $\mu$ follow the PY values not only at low $\rho$ but throughout the entire range of densities; we, therefore, fix these parameters at their PY values. We remind, in passing, that a similar finding regarding the decay parameters $\kappa$ and $\omega$ was noted recently also by Roth et $a l_{.}{ }^{22}$ who studied the correlations of a confined HS fluid. By comparing the density-functional theory approach to the HS problem with corresponding MC 


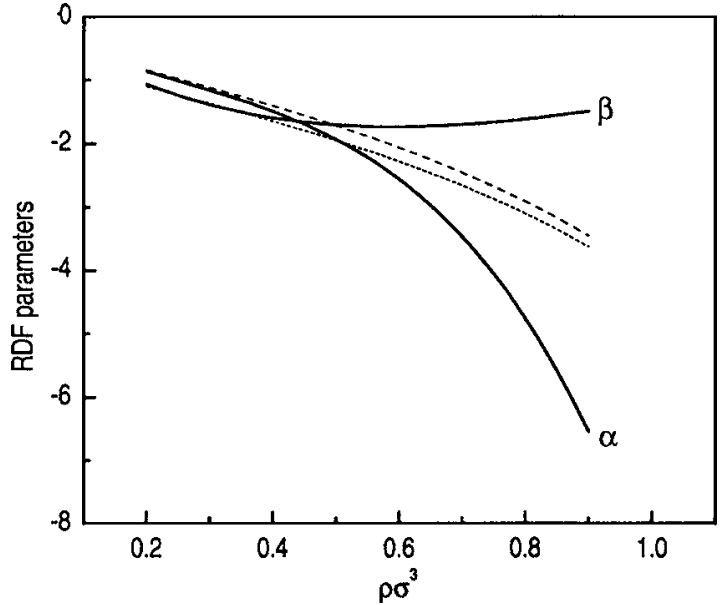

FIG. 2. Parameter $\alpha \sigma$ and $\beta \sigma$ (solid lines) of the parametrized hard-sphere RDF [Eqs. (14)-(16)] and the corresponding PY values (dashed lines).

data, they obtained an accurate fit for the parameters $\omega_{o}$ and $\kappa_{o}$. Combining our finding with the results of Roth et al., ${ }^{22}$ we can write

$$
\omega \sigma \equiv \omega_{o} \sigma=-0.682 \exp (-24.697 \eta)+4.720+4.450 \eta,
$$

$$
\begin{aligned}
\kappa \sigma \equiv \kappa_{o} \sigma= & 4.674 \exp (-3.935 \eta) \\
& +3.536 \exp (-56.270 \eta)
\end{aligned}
$$

Parameters $\mu_{\mathrm{o}}$ and $\gamma_{\mathrm{o}}$ can be evaluated directly using the original Wertheim solution, ${ }^{5}$

$$
\begin{aligned}
\mu \sigma \equiv \mu_{o} \sigma=\frac{2 \eta}{1-\eta}\left(-1-\frac{d}{2 \eta}-\frac{\eta}{d}\right) \\
\gamma \equiv \gamma_{o}=\arctan \left\{-\frac{\sigma}{\beta_{o}}\left[\left(\alpha_{o} \sigma\left(\alpha_{o}^{2}+\beta_{o}^{2}\right)-\mu_{o} \sigma\left(\alpha_{o}^{2}+\beta_{o}^{2}\right)\right)\right.\right. \\
\left.\left.\times\left(1+\frac{1}{2} \eta\right)+\left(\alpha_{o}^{2}+\beta_{o}^{2}-\mu_{o} \alpha_{o}\right)(1+2 \eta)\right]\right\},(30)
\end{aligned}
$$

where

$$
\begin{aligned}
& \alpha_{o} \sigma=\frac{2 \eta}{1-\eta}\left(-1+\frac{d}{4 \eta}-\frac{\eta}{2 d}\right), \\
& \beta_{o} \sigma=\frac{2 \eta}{1-\eta} \sqrt{3}\left(-\frac{d}{4 \eta}-\frac{\eta}{2 d}\right),
\end{aligned}
$$

with

$$
d=\left[2 \eta\left(\eta^{2}-3 \eta-3+\sqrt{3\left(\eta^{4}-2 \eta^{3}+\eta^{2}+6 \eta+3\right)}\right)\right]^{1 / 3} .
$$

There are therefore only three parameters, $\alpha, \beta$, and $r^{*}$ as well as $g_{m}$ to be determined from Eqs. (17)-(20). Resulting values of $\alpha$ and $\beta$ are plotted in Fig. 2. As expected, both parameters approach the PY results (31) at low densities. However, as the density increases, $\alpha$ and $\beta$ start to deviate from their PY values and, approximately for $\rho \sigma^{3}>0.4$, they exhibit a density behavior that is totally different from that of
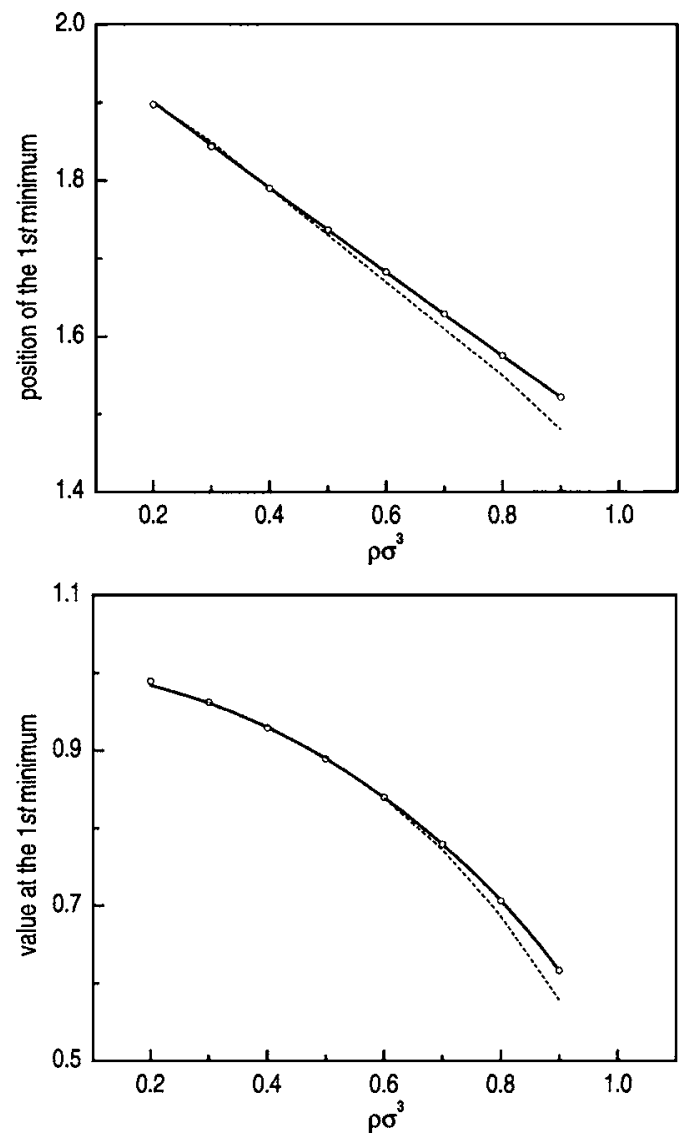

FIG. 3. Position $r_{m} / \sigma$ and the value $g_{m}$ of the first minima of the hardsphere RDF obtained from the solution of Eqs. (17)-(20). The symbols are MC data and the dashed lines show the corresponding PY values.

their PY originals, $\alpha_{\mathrm{o}}$ and $\beta_{\mathrm{o}}$. We find that in the range of densities $0.2 \leqslant \rho \sigma^{3} \leqslant 0.9$, the parameters $\alpha$ and $\beta$ can be accurately parametrized in the form.

$$
\begin{aligned}
& \alpha \sigma=44.554+79.868 \eta+116.432 \eta^{2}-44.652 \exp (2 \eta), \\
& \beta \sigma=-5.022+5.857 \eta+5.089 \exp (-4 \eta) .
\end{aligned}
$$

For the densities, $\rho \sigma^{3}<0.2$, the parameters $\alpha$ and $\beta$ should be replaced by their PY values given by Eq. (31). The stronger and distinct dependence on the density of $\alpha$ and $\beta$ in comparison to that of $\alpha_{o}$ and $\beta_{o}$ can be understood after analyzing the density dependence of the RDF minimum position $r_{m}$ and the minimum value $g_{m}$. These data are shown in Fig. 3 and include the results from the PY theory and MC simulations. The first things we note are the different trends that the values of $r_{m}^{\mathrm{PY}}$ and $g_{m}^{\mathrm{PY}}$ exhibit at low and high densities with respect to the MC data. In particular, in the density region $0.2<\rho \sigma^{3}<0.4$, the position of the first minimum of $g^{\mathrm{PY}}(r)$ is systematically shifted towards slightly larger separations than follows from computer simulations. However, this trend is reversed when the density increases, as is clearly seen from Fig. 3 for $\rho \sigma^{3}>0.5$. A similar density behavior is exhibited by the magnitude $g_{m}^{\mathrm{PY}}$ of the first minimum of $g^{\mathrm{PY}}(r)$. It is then obvious that some parameters in the functional expression of $g(r)$ must be distinct from their PY analogs to account for such density features of $g^{\mathrm{PY}}(r)$. 

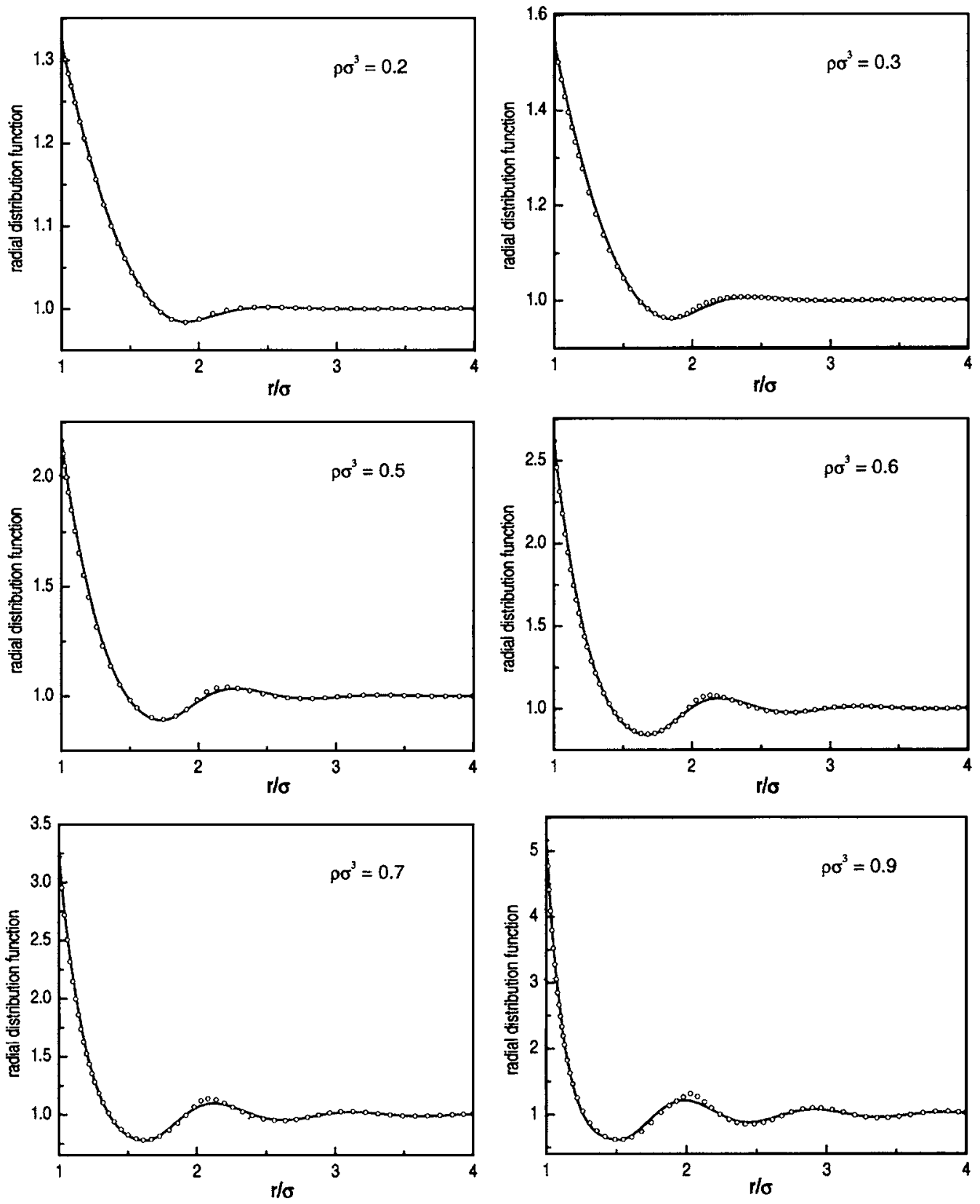

FIG. 4. The hard-sphere RDF evaluated using Eqs. (14)-(16) (thin solid line) and the corresponding MC data (symbols) at different densities.

The position $r^{*} \equiv r_{m}$ of the RDF first minimum is one of the key ingredients in the proposed equation (14) for $g(r)$. The parameter $r^{*}$ and the value of the first minimum $g_{m}$ both have been found as the outcome of a self-consistent solution of the Eqs. (17)-(20) and are shown in Fig. 3. For convenience, the results for $r^{*} \equiv r_{m}$ and $g_{m}$ have been parametrized over the range of densities $0.2 \leqslant \rho \sigma^{3} \leqslant 0.9$ in the form,

$$
\begin{aligned}
r^{*} / \sigma & \equiv r_{m} / \sigma=2.0116-1.0647 \eta+0.0538 \eta^{2}, \\
g_{m}= & 1.0286-0.6095 \eta+3.5781 \eta^{2}-21.3651 \eta^{3} \\
& +42.6344 \eta^{4}-33.8485 \eta^{5} .
\end{aligned}
$$

As one can see in Fig. 3, the results obtained from our procedure are in an excellent agreement (within the simulation errors) with the simulation data.

\section{DISCUSSION AND CONCLUSIONS}

Figure 4 presents a set of results for the hard-sphere RDF evaluated using Eqs. (14)-(16). The results cover the density range from $\rho \sigma^{3}=0.2$ up to 0.9 and are compared with the latest high accuracy MC data. ${ }^{3}$

In general, the overall agreement between the proposed analytic representation of $g(r)$ and simulation data is very good for all $r$ 's and densities, but not perfect in every region. The general trend is easy to detect: in the region where the two different forms are fused together, a discrepancy exceeding experimental errors is observed with increasing density. This is quite understandable if we realize that the proposed $g(r)$ is built using concepts from liquid-state theory. It is known that as the freezing density is approached, the RDF starts to exhibit a typical shoulder which indicates beginning of the formation of amorphouslike or crystallinelike struc- 

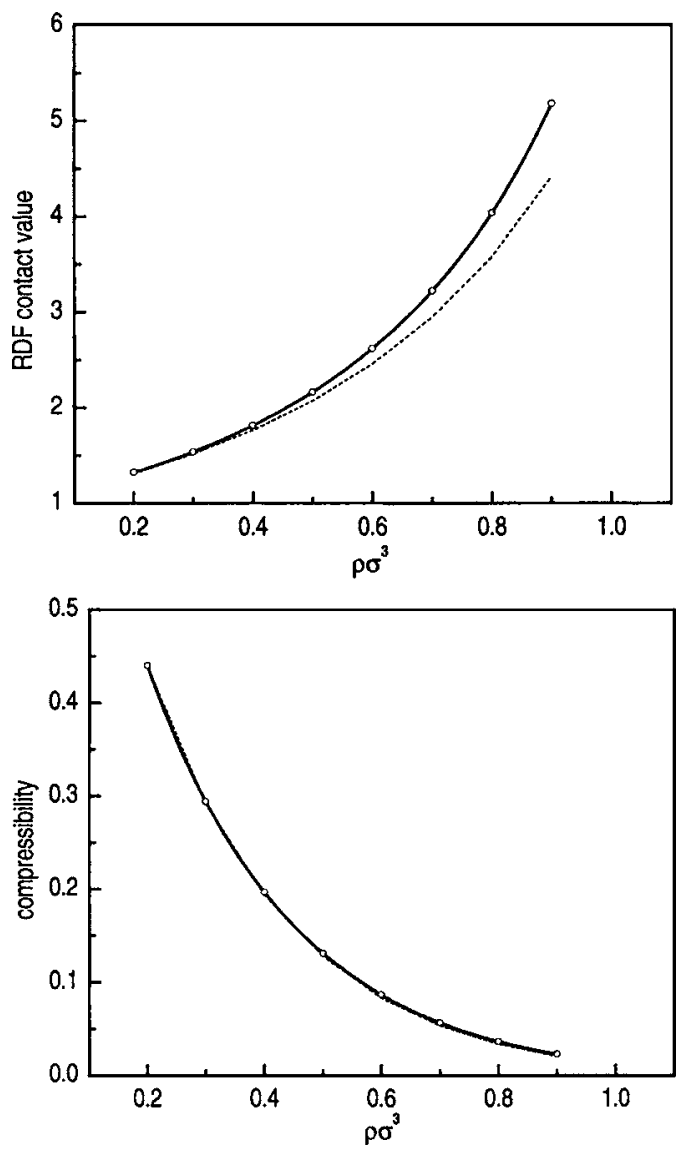

FIG. 5. Contact values of the RDF and the isothermal compressibility of the hard-sphere fluid that follow from the RDF given by Eqs. (14)-(16) (solid lines) and MC data (symbols). The dashed lines show the corresponding PY results.

tures and this phenomenon (the occurrence of islands of such structures) cannot be, in principle, described by $g(r)$ given by Eq. (14).

Finding the parameters of an analytic expression of $g(r)$ by imposing certain constraints does not necessary mean that such constraints are exactly satisfied. The discussed solution has been found by minimizing an appropriate functional and the question is to what accuracy are Eqs. (17)-(20) satisfied. The results are summarized in Fig. 5. First of all, we note that the condition for the contact value of $g(r)$ is fully satisfied. This is very important because for HS fluid the value of $g(r)$ at $r=\sigma$ determines the pressure in the system. As for the compressibility $K_{T}$, this is a more subtle property with which to verify the accuracy of $g(r)$ because, according to the definition (18), it is related to the integral of $g(r)$. Nevertheless, we can claim that our results follow the simulation data, ${ }^{3}$ which in turn indicates that the system pressure $p$, being evaluated from the isothermal compressibility, would also be correct. To summarize, the accurate results for both the contact value and compressibility guarantee the thermodynamic self-consistency of the proposed equation for $g(r)$. In this context we recall that the thermodynamic consistency has been the major problem of the RDF obtained within the PY theory. Indeed, we can see from Fig. 5 that the PY compressibility, $K_{T}^{\mathrm{PY}}=(1-\eta)^{4} /(1+2 \eta)^{2}$, that follows from $g^{\mathrm{PY}}(r)$, agrees reasonably well with the simulation data. However,

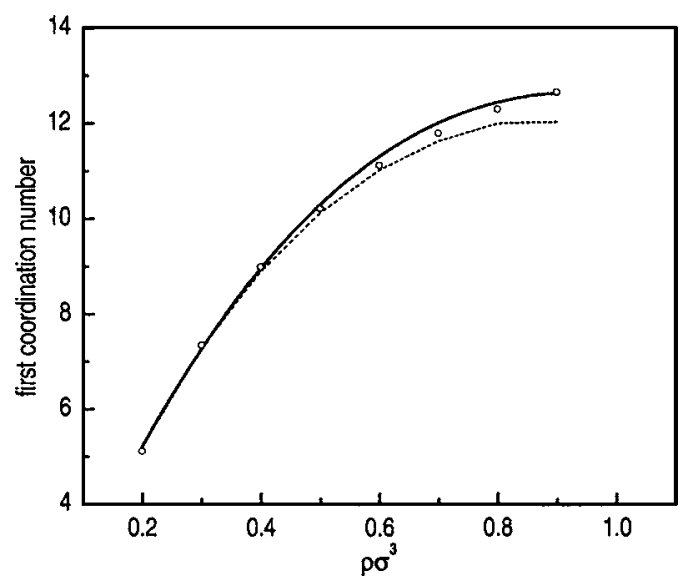

FIG. 6. The same as Fig. 5 for the first coordination number of the hardsphere fluid.

this result is inconsistent with the PY result for the contact value, $g_{\sigma}^{\mathrm{PY}}=\left(1+\frac{1}{2} \eta\right) /(1-\eta)^{2}$, which disagrees with the exact data. This problem of the PY theory for HS fluid is well known and has been discussed in detail elsewhere. ${ }^{11}$

From another point of view, with respect to the twopiecewise character of $g(r)$, the isothermal compressibility can also be treated as a composition of two contributions that arise from the depletion and structural parts of $g(r)$, i.e.,

$$
\begin{aligned}
K_{T}= & 1+4 \pi \rho \int_{0}^{\infty}(g(r)-1) r^{2} d r \\
= & 1-8 \eta r_{m}^{3}+4 \pi \rho \int_{\sigma}^{r_{m}} g^{\operatorname{dep}}(r) r^{2} d r \\
& +4 \pi \rho \int_{r_{m}}^{\infty}\left(g^{\operatorname{str}}(r)-1\right) r^{2} d r,
\end{aligned}
$$

where the integral associated with $g^{\mathrm{dep}}(r)$ is the coordination number,

$$
n_{1}=4 \pi \rho \int_{\sigma}^{r_{m}} g^{\mathrm{dep}}(r) r^{2} d r .
$$

From the results shown in Fig. 6 we may conclude that the PY theory fails not only at the contact $r=\sigma$, but that the entire profile of $g^{\mathrm{PY}}(r)$ for $\sigma<r<r_{m}$ is not correct and this results in disagreement of $n_{1}^{\mathrm{PY}}$ with $n_{1}^{\text {expt }}$ from MC data. This means that for $K_{T}^{\mathrm{PY}}$ to be in fair agreement with the simulation data, the remaining integral of $g^{\mathrm{PY}}(r)$ in Eq. (37), namely, integral associated with the structural part of $g^{\mathrm{PY}}(r)$, must also deviate from the exact value to compensate for errors in the coordination number $n_{1}^{\mathrm{PY}}$.

Besides the thermodynamic self-consistency discussed above, in the case of HS fluid there are other theoretically imposed conditions that involve the RDF, primarily the wellknown zero-separation theorems ${ }^{16-18}$ for the correlation functions at distance $r=0$, i.e., when two hard spheres coincide. Following Lee and Shing ${ }^{23}$ a first zero-separation theorem $^{24-27}$ (ZST1) can be written for the direct correlation function, $-c(0)=1 / K_{T}+I_{\mathrm{LM}}$, and examined numerically in the form, 


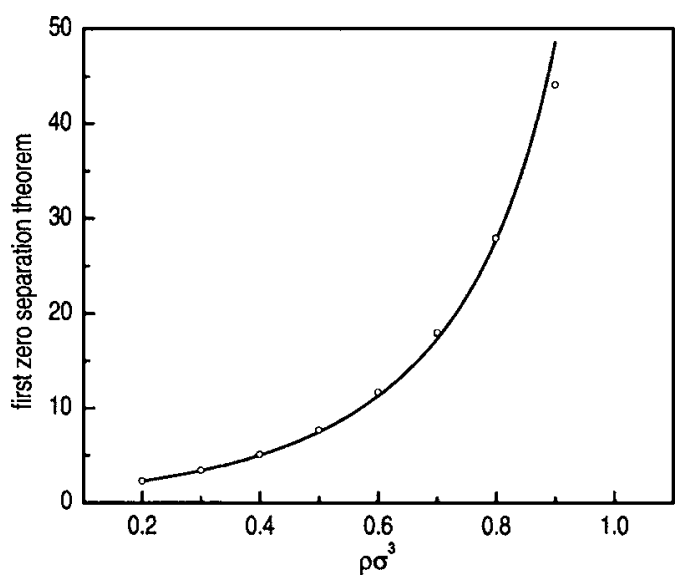

FIG. 7. The same as Fig. 5 for the first zero-separation theorem.

$$
\frac{-1}{2 \pi^{2}} \int_{0}^{\infty} \frac{\tilde{h}(k)}{1+\rho \widetilde{h}(k)} k^{2} d k=\frac{1}{K_{T}}+I_{\mathrm{LM}},
$$

where

$$
I_{\mathrm{LM}} \equiv 4 \pi \rho \int_{0}^{\infty} g(r) c(r) r^{2} d r=\frac{\eta^{3}}{(1-\eta)^{6}}[1.805-3.512 \eta]
$$

is the result obtained by Malijevský and Labík, ${ }^{12}$ and

$$
\widetilde{h}(k)=4 \pi \int_{0}^{\infty}[g(r)-1] \frac{\sin (k r)}{k r} r^{2} d r .
$$

The integral in Eq. (41) can be evaluated analytically and the ZST1 in the form of Eq. (39) is expressed in terms of the parameters of $g(r)$. The corresponding results are presented in Fig. 7 showing a close agreement between the calculated and expected data over all considered densities. This illustrate, in particular, that $g(r)$ given by Eq. (14) will provide with an accurate result for the DCF of the HS fluid at $r=0$. Some visible discrepancy between our calculations and the expected values around the highest density. $\rho \sigma^{3}=0.9$ in Fig. 7 could result from the both numerical evaluation of the integral in Eq. (39) as well as inaccuracy of the Malijevský and Labík expression (40) at this density.

There are no exact relations that involve the values of DCF at other specified distances $r$ and that could be used to examine the accuracy of $g(r)$. An important quantity for the HS fluid is the contact distance $r=\sigma$, where both $g(r)$ and $c(r)$ exhibit discontinuities. This provides one only with an exact value of the discontinuity jump $c\left(r=\sigma^{+}\right)-c\left(r=\sigma^{-}\right)$ $=g_{\sigma}$, while the values $c\left(\sigma^{+}\right)=g_{\sigma}-1+\gamma(\sigma)$ and $c\left(\sigma^{-}\right)=-1$ $+\gamma(\sigma)$ are not certain. However, we note that the function $\gamma(r=\sigma)$, [see Eq. (3)] is continuous and can be evaluated numerically, since function $\tilde{h}(k)$ is known, using $\tilde{\gamma}(k)$ $=\rho \widetilde{h}^{2}(k) /(1+\rho \tilde{h}(k))$. Thus, some approximate values of $c\left(\sigma^{+}\right)$and $c\left(\sigma^{-}\right)$can be calculated numerically.

The second zero-separation theorem (ZST2) concerns the derivative of $c(r)$ at $r \rightarrow 0 .{ }^{27}$ However, using any conceivable analytic continuation, the derivatives of $c(r)$ for the HS fluid are not continuous at $r=0$; this prevents $d c(r) / d r$ at $r=0$ from being correctly evaluated numerically from the Fourier transform $\tilde{h}(k)$. For this reason, we do not exploit the ZST2 in this paper.

Summarizing, we present a closed-form analytical equation (14) for the radial distribution function $g(r)$ of hardsphere fluid. The proposed equation consists of two parts, $g^{\mathrm{dep}}(r)$ and $g^{\mathrm{str}}(r)$, of different functional forms that correspond for short and large distances, respectively. Both parts of Eq. (14) are merged at a distance $r=r^{*}$ where $g(r)$ exhibits the first minimum. The different functional forms of $g(r)$ for short and large distances reflect the distinction of two-body and many-body nature of the interparticle correlations contributing to $g(r)$ and are referred here to as the depletion and structural parts of the RDF. The term "depletion" means that there is the exclusion of the spheres mediators from the gap between a pair of selected spheres that, indeed, takes a place for the short distances up to the position of the first minimum in $g(r)$, i.e., between a central sphere and its nearest neighbors. In contrast, the term "structural" refers to the particleparticle separations when there are other spheres in a space between a pair of selected spheres that usually appear for distances larger than the position of the first minimum of $g(r)$, i.e., between a central sphere and those beyond the nearest-neighbor shell. Both the functional forms (15) and (16) that describe the depletion and structural contributions have been obtained from the known solutions (13) and (11), respectively, of the Percus-Yevick equation. In this way a purely empirical fit of the computer simulation data is avoided. Both parts of Eq. (14) involve altogether 11 parameters, including one for the merging distance, $r^{*}$, and have been determined by forcing $g(r)$ to assume the experimentally established value at the contact distance $r=\sigma$ and fulfill the thermodynamic consistency condition (18). In fact, proceeding in this way, we found that only 7 of the 11 parameters are independent and need be obtained by solving Eqs. (17)-(20). Moreover, by minimizing an appropriate functional we found that some of these parameters take their PY values, known from the Wertheim solution, ${ }^{5}$ while the remaining have been accurately parametrized. The complete scheme of calculation is summarized in the Appendix.

The proposed analytic representation (14) of $g(r)$ is valid for all distances $r$ and densities $\rho$ in the range $0.2 \leqslant \rho \sigma^{3}$ $\leqslant 0.9$. For densities $\rho \sigma^{3}$ less than 0.2 , the RDF of the HS fluid switches its shape from oscillatory to monotonic. The latter means that in Eq. (7) the poles with only real part becomes important. Consequently, solutions (11) and (13) simplify by consisting only exponentials terms; the resulting $g(r)$ will not exhibit anymore the minimum position $r_{m}$. Because of this, the density region $\rho \sigma^{3}<0.2$ must be treated separately. This can be done within the present scheme by using another choice for the merging distance (e.g., assuming that the merging distance is at the end of the first distance zone, i.e., $r^{*}=2 \sigma$ ) or applying the results obtained within the density expansion theory for the HS fluid. ${ }^{28}$

The RDF evaluated using Eq. (14) shows a very good overall agreement with the simulation data. The discrepancies in the profile of $g(r)$ that exceed the experimental errors are observed with increasing density and for the narrow (around a half of sphere diameter) distance region bounded 
by the positions of the first minimum and the second maximum, just after the distance at which the depletion and structural parts of $g(r)$ are fused together. In general, there is the way to fight these discrepancies by including more terms to represent the structural part of $g(r)$. Each new terms will be of the same functional form as that of the existing term (16), describing the contributions to $g(r)$ from the residues (10) of the remaining poles in Eq. (7). Eventually, this will complicate the final equation for $g(r)$ while our aim is to keep this equation as simple as possible. On the other hand, the character of the discrepancies due to the simplicity of Eq. (14) is that they are partially self-canceling in the integrals of $g(r)$ of the type (37) that are used to evaluate the compressibility. A close examination of some other properties involving the profile of $g(r)$ shows that the consequences of these discrepancies are negligible; for example, in the calculations of coordination number $n_{1}$ and direct correlation function $c(r$ $=0)$. At the same time we stress that $g(r)$ given by Eq. (14) cannot be used for densities $\rho \sigma^{3}$ greater than 0.9 since it has been built using concepts from liquid-state theory. At the densities beyond 0.9 , where roughly the random close packing region starts, the HS fluid RDF exhibits a typical shoulder which indicates beginning of the formation of amorphouslike or crystallinelike structures and this phenomenon (the occurrence of islands of such structures) cannot be, in principle, described by $g(r)$ given by simple Eq. (14).

Concluding, the analytical equation (14) for the RDF should be useful in various applications of the HS fluid in the density range $0.2 \leqslant \rho \sigma^{3} \leqslant 0.9$. Moreover, having such an analytical expression for $g(r)$ that consists of explicit depletion and structural contributions can be useful to help to understand the impact of each of these parts of RDF on the other properties evaluated by using $g(r)$. From other perspective, our pursued strategy can be applied to obtain RDF of the highly size asymmetric binary HS mixture where two different length scales related with the diameters of the large and small spheres are present. Additionally, those who are working in developing approximate theories for HS fluid can employ the present $g(r)$ to calculate the bridge function for distances $r \geqslant \sigma$. However, without a knowledge of the cavity distribution function $y(r)=\exp [\beta u(r)] g(r)$ for $r<\sigma$, one cannot obtain the bridge function for whole range of distances.

\section{ACKNOWLEDGMENTS}

This research was supported by the "Information Society" research program (Project No. 1ET400720409), by the EU project BEMUSAC (Contract No. G1MA-CT-200204019), and by the National Science Foundation (Grant No. CTS-9704044).

\section{APPENDIX: A RECIPE TO CALCULATE ACCURATE RDF OF A HARD-SPHERE FLUID}

To evaluate the RDF of the hard-sphere fluid for a given density $\rho \sigma^{3}$ or packing fraction $\eta=\pi \rho \sigma^{3} / 6$ first one has to calculate the parameters obtained by Wertheim ${ }^{5}$ from the solution of the PY equation,

$$
\begin{aligned}
\mu \sigma= & \frac{2 \eta}{1-\eta}\left(-1-\frac{d}{2 \eta}-\frac{\eta}{d}\right), \\
\gamma= & \arctan \left\{-\frac{\sigma}{\beta_{o}}\left[\left(\alpha_{o} \sigma\left(\alpha_{o}^{2}+\beta_{o}^{2}\right)-\mu_{o} \sigma\left(\alpha_{o}^{2}+\beta_{o}^{2}\right)\right)\right.\right. \\
& \left.\left.\times\left(1+\frac{1}{2} \eta\right)+\left(\alpha_{o}^{2}+\beta_{o}^{2}-\mu_{o} \alpha_{o}\right)(1+2 \eta)\right]\right\},
\end{aligned}
$$

where

$$
\begin{aligned}
& \alpha_{o} \sigma=\frac{2 \eta}{1-\eta}\left(-1+\frac{d}{4 \eta}-\frac{\eta}{2 d}\right) \\
& \beta_{o} \sigma=\frac{2 \eta}{1-\eta} \sqrt{3}\left(-\frac{d}{4 \eta}-\frac{\eta}{2 d}\right), \\
& d=\left[2 \eta\left(\eta^{2}-3 \eta-3+\sqrt{3\left(\eta^{4}-2 \eta^{3}+\eta^{2}+6 \eta+3\right)}\right)\right]^{1 / 3} .
\end{aligned}
$$

Next are the coefficients parametrized by Roth et al., ${ }^{22}$

$$
\begin{aligned}
& \omega \sigma=-0.682 \exp (-24.697 \eta)+4.720+4.450 \eta, \\
& \kappa \sigma=4.674 \exp (-3.935 \eta)+3.536 \exp (-56.270 \eta),
\end{aligned}
$$

and those parametrized in present study,

$$
\begin{aligned}
\alpha \sigma= & 44.554+79.868 \eta+116.432 \eta^{2}-44.652 \exp (2 \eta), \\
\beta \sigma & =-5.022+5.857 \eta+5.089 \exp (-4 \eta), \\
r^{*} / \sigma= & 2.0116-1.0647 \eta+0.0538 \eta^{2}, \\
g_{m}= & 1.0286-0.6095 \eta+3.5781 \eta^{2}-21.3651 \eta^{3} \\
& +42.6344 \eta^{4}-33.8485 \eta^{5} .
\end{aligned}
$$

After this, the remaining parameters can be expressed in the terms of known parameters,

B

$$
\begin{gathered}
=\frac{g_{m}-\left(\sigma g_{\sigma}^{\operatorname{expt}} / r^{*}\right) \exp \mu\left[r^{*}-\sigma\right]}{\cos \left(\beta\left[r^{*}-\sigma\right]+\gamma\right) \exp \alpha\left[r^{*}-\sigma\right]-\cos \gamma \exp \mu\left[r^{*}-\sigma\right]} r^{*}, \\
A=\sigma g_{\sigma}^{\operatorname{expt}}-B \cos \gamma, \\
\delta=-\omega r^{*}-\arctan \frac{\kappa r^{*}+1}{\omega r^{*}}, \\
C=\frac{r^{*}\left[g_{m}-1\right] \exp \kappa r^{*}}{\cos \left(\omega r^{*}+\delta\right)},
\end{gathered}
$$

where

$$
g_{\sigma}^{\text {expt }}=\frac{1}{4 \eta}\left(\frac{1+\eta+\eta^{2}-2 / 3 \eta^{3}-2 / 3 \eta^{4}}{(1-\eta)^{3}}-1\right) .
$$

Finally, the RDF of the hard-sphere fluid in the range of densities $0.2 \leqslant \rho \sigma^{3} \leqslant 0.9$ has the form,

$g(r)=0$ for $r<\sigma$ 


$$
\begin{aligned}
& =\frac{A}{r} e^{\mu[r-\sigma]}+\frac{B}{r} \cos (\beta[r-\sigma]+\gamma) e^{\alpha[r-\sigma]} \quad \text { for } \sigma \leqslant r \leqslant r^{\star} \\
& =1+\frac{C}{r} \cos (\omega r+\delta) e^{-\kappa r} \quad \text { for } r \geqslant r^{\star}
\end{aligned}
$$

${ }^{1}$ T. Boublík and I. Nezbeda, Collect. Czech. Chem. Commun. 51, 2301 (1986).

${ }^{2}$ I. Nezbeda, S. Labík, and A. Malijevský, Collect. Czech. Chem. Commun. 54, 1137 (1989).

${ }^{3}$ J. Kolafa, S. Labík, and A. Malijevský, Phys. Chem. Chem. Phys. 6, 2335 (2004).

${ }^{4}$ S. Labík, J. Kolafa, and A. Malijevský, Phys. Rev. E 71, 021105 (2005).

${ }^{5}$ M. S. Wertheim, Phys. Rev. Lett. 10, 321 (1963).

${ }^{6}$ E. Thiele, J. Chem. Phys. 39, 474 (1963).

${ }^{7}$ W. R. Smith and D. J. Henderson, Mol. Phys. 19, 411 (1970).

${ }^{8}$ I. Nezbeda, Czech. J. Phys., Sect. B 24, 55 (1974).

${ }^{9}$ J. Perram, Mol. Phys. 30, 1505 (1975).

${ }^{10}$ J. Chang and S. I. Sandler, Mol. Phys. 81, 735 (1994).
${ }^{11}$ L. Verlet and J. J. Weiss, Phys. Rev. A 5, 939 (1972).

${ }^{12}$ A. Malijevský and S. Labík, Mol. Phys. 67, 431 (1989).

${ }^{13}$ J. P. Hansen and I. R. McDonald, Theory of Simple Liquids (Academic, New York, 1986).

${ }^{14}$ L. Verlet, Mol. Phys. 41, 183 (1980).

${ }^{15}$ G. A. Martynov and G. N. Sarkisov, Mol. Phys. 49, 1495 (1983).

${ }^{16}$ L. L. Lee, J. Chem. Phys. 97, 8606 (1992).

${ }^{17}$ L. L. Lee, J. Chem. Phys. 103, 9388 (1995).

${ }^{18}$ L. L. Lee, J. Chem. Phys. 110, 7589 (1999).

${ }^{19}$ J. A. Barker and D. Henderson, Rev. Mod. Phys. 48, 587 (1976)

${ }^{20}$ P. Perry and G. J. Throop, J. Chem. Phys. 57, 1827 (1972).

${ }^{21}$ W. H. Press, S. A. Teukolsky, W. T. Vetterling, and B. P. Flannery, $N u-$ merical Recipes in Fortran 77, 2nd ed. (Cambridge University Press, Cambridge, 2003)

${ }^{22}$ R. Roth, R. Evans, and S. Dietrich, Phys. Rev. E 62, 5360 (2000).

${ }^{23}$ L. L. Lee and K. S. Shing, J. Chem. Phys. 91, 477 (1989).

${ }^{24}$ E. Meeron and A. J. F. Siegert, J. Chem. Phys. 48, 3139 (1968).

${ }^{25}$ E. W. Grundke and D. Henderson, Mol. Phys. 24, 269 (1972).

${ }^{26}$ B. Barboy and R. Tenne, Mol. Phys. 31, 1749 (1976).

${ }^{27}$ Y. Zhou and G. Stell, J. Stat. Phys. 52, 1389 (1988).

${ }^{28}$ B. R. A. Nijboer and L. Van Hove, Phys. Rev. 85, 777 (1952). 